\title{
Goal-directed and stimulus-driven attention in cross-dimensional texture segregation
}

\author{
THOMAS G. GHIRARDELLI and HOWARD E. EGETH \\ Johns Hopkins University, Baltimore, Maryland
}

\begin{abstract}
Goal-directed and stimulus-driven control of attention was examined in a visual texture segregation task. Recent published reports have debated the existence and efficiency of goal-directed guidance of attention. Some of this research has focused on the apparent stimulus-driven attentional priority given to salient distractors, even when they are known to be irrelevant to the task. In the present study, subjects searched a texture array for targets defined along one dimension. These displays also included distractors created by variation in an irrelevant dimension. Targets were of three different overall shapes. On each trial, distractors could be the same shape as the target or one of the other two shapes. In two experiments subjects were informed of the overall shape of the target prior to stimulus presentation. In these experiments, distractors that did not match the overall shape of the target caused less interference than distractors that matched the target's shape. In the third experiment, subjects were not informed of the overall shape of the target. In this experiment all distractors caused roughly equal interference. The results of these experiments demonstrate that if subjects are given information about the overall shape of the target, they are able to use this information to reduce interference from distractors that do not match the overall target shape. While acknowledging some stimulus-driven interference, this illustrates a previously unexplored source of goal-directed guidance that can reduce interfering effects of even salient distractors and argues against purely stimulus-driven control of attention.
\end{abstract}

Several recent theories of visual information processing suggest that spatial attention is controlled by two factors. The existence of two separate mechanisms of attentional control was first proposed by James (1890) and further elucidated by Broadbent (1958). Yantis (1993a) has referred to these two modes of control as stimulus-driven capture and goal-directed selection. Stimulus-driven capture (also called bottom-up control) is said to occur when the observer's attention is drawn to a stimulus independent of the observer's intent, goals, or expectations. Goaldirected selection (also called top-down control) is said to occur when the observer's knowledge, expectations, and intentional state determine what stimulus is selected.

One piece of evidence for the importance of goaldirected information comes from studies using spatial cuing tasks. Posner, Snyder, and Davidson (1980) showed that the presence of a centrally presented symbolic cue (a

\footnotetext{
The experiments reported here formed part of the doctoral dissertation submitted to Johns Hopkins University by T.G.G. This research was supported in part by NSF Grant SBR-9319356 and NIH Grant R01 MH57388 awarded to H.E.E., and NIH Grant 5 T32 MH18125, awarded to the Department of Psychology at Johns Hopkins University. We thank Chip Folk for helpful discussion of this work and Kyle Cave, Art Kramer, Roger Remington, and an anonymous reviewer for thoughtful comments on an earlier version of this paper. Preparation of this manuscript was completed while T.G.G. held an IRTA postdoctoral fellowship at the National Institute on Alcoholism and Alcohol Abuse (NIAAA). Portions of this work were presented at the thirty-sixth annual meeting of the Psychonomic Society, Los Angeles. Correspondence should be addressed to T. G. Ghirardelli, NIAAA, 10 Center Dr., Room 6S240, MSC 1610, National Institutes of Health, Bethesda, MD 20892-1610 (e-mail: ghirardelli@nih.gov).
}

digit that indicated one of four possible target locations) enabled subjects to respond to targets more rapidly when the cue indicated the correct subsequent target location (i.e., when it was a valid cue) and caused them to respond more slowly when it indicated an incorrect location (i.e., when it was an invalid cue). This cue represents a goaldirected source of information because the cue itself does not provide any information about the target, or the correct response, directly (e.g., via its location or brightness) but had to be interpreted by the subjects. Subjects voluntarily allocated attention to the cued location, resulting in benefits in response time (RT) for valid cues and costs in RT for invalid cues.

Yantis and Jonides (1984) used a visual search task to demonstrate that stimulus-driven information also has a powerful effect on attention. Subjects searched for a specified letter target in a display of up to four letters. All but one of the letters in each display were revealed by removing line segments from placeholders that appeared at the start of the trial. One letter on each trial appeared as an abrupt onset, where no placeholder had appeared. The abrupt onset letter was no more likely to be the target than any other letter, and thus the information provided by the onset was uninformative. Nonetheless, for trials in which the target was not the onset letter, the amount of time it took subjects to find the target increased with an increase in the number of letters in the display. The number of letters in the display had no effect on the time it took to find the target when the target was the onset letter, suggesting that the onset letter was always the first letter attended. Yantis and Jonides concluded that because 
the onset letter was not related to the target, this letter captured attention in a stimulus-driven manner.

There is also considerable evidence for the joint operation of both goal-directed and stimulus-driven information in visual information processing using visual search tasks (see, e.g., Bacon \& Egeth, 1994; Kaptein, Theeuwes, \& Van der Heijden, 1995; Poisson \& Wilkinson, 1992) and a modified spatial cuing task (see, e.g., Folk \& Remington, 1998; Folk, Remington, \& Johnston, 1992; Folk, Remington, \& Wright, 1994). Many of the studies designed to examine goal-directed and stimulus-driven guidance of attention have utilized methodologies that pit these two modes of control against each other. One way of accomplishing this is by presenting singletons defined on one dimension as targets and singletons defined on another dimension - that is known to be irrelevant to the task - as distractors. Subjects' awareness of the targetdefining dimension represents a goal-directed source of guidance. Even when singletons are known to be irrelevant, and thus provide no goal-directed guidance, they may capture attention solely on the basis of the stimulusdriven information they provide (see, e.g., Bacon \& Egeth, 1994; Joseph \& Optican, 1996; Theeuwes, 1992). Many studies have suggested that singletons receive priority in processing because initial (preattentive) processing of visual displays detects areas of difference and therefore may respond preferentially to singletons as regions of high dissimilarity (e.g., Bergen \& Julesz, 1983; Pashler, 1988). This is consistent with many models of visual search, such as Guided Search 2.0 (GS2; Wolfe, 1994). There remains some debate over the existence and efficiency of goal-directed guidance of attention, however (see, e.g., Folk, Remington, \& Johnston, 1993; Theeuwes, 1994; Yantis, 1993b).

In one study pitting goal-directed and stimulus-driven guidance against each other, Folk et al. (1992; see also Folk et al., 1994) had subjects search for a target singleton among three other characters. The target was defined on one dimension (e.g., color) and subjects had to determine if the colored (e.g., red) item was an "X" or an "=." The characters appeared in four boxes arranged on an imaginary cross centered on fixation. Before the target display, a distractor display was presented. This distractor display consisted of markers surrounding each of the four boxes. In the important conditions, one set of markers differed from the other three on a particular dimension (i.e., that set formed an irrelevant singleton). Folk et al. (1992) found that this distractor display interfered with subjects' performance when the singleton distractor was spatially incompatible with the subsequent target, but only if the distractor was defined along the same dimension as the target. For example, if the target was defined as a color singleton, a colored distractor at a different location interfered, but a distractor defined on a different dimension (e.g., an abrupt onset) did not. This result formed the basis of their contingent involuntary orienting hypothesis. According to this hypothesis, singleton distractors will capture attention only when they share a feature property that is critical to performing the task at hand. Thus, goal-directed information can influence the stimulus-driven information provided by even a salient item.

It has been suggested that the asynchronous presentation of the singleton distractor and singleton target in the study by Folk et al. (1992) may have made the interpretation of their results open to alternatives (Yantis, 1993b; but see Folk et al., 1993). In a study using the simultaneous presentation of singleton targets and distractors, Theeuwes (1991) used a visual search task to assess whether goaldirected information about a particular stimulus dimension could override the stimulus-driven capture of a singleton in a different, irrelevant dimension. He presented five, seven, or nine colored shapes equally spaced around the fixation point on an imaginary circle. The target was a unique shape (e.g., a circle among diamonds or a diamond among circles). Subjects made a forced-choice response (horizontal or vertical) to a line segment presented within the target shape. Oblique line segments were presented in the distractor shapes. When one of the distractors was a color singleton (i.e., presented in red among green shapes), RT increased, suggesting that the presence of an irrelevant but unique color singleton slows down search for a unique form. Because the color difference was more salient than the form difference, Theeuwes suggested that the color singleton was selected first, even though color was known to be irrelevant to the task. In a subsequent series of experiments, Theeuwes (1992) showed that specifying the exact feature value of the target (e.g., not just the odd shape, but the green circle) did not reduce the interfering effects of the color singleton. Even when subjects were provided with this additional information, the presence of an irrelevant color singleton increased RTs. Theeuwes used this result to argue that selection is never goal directed, that, in fact, selection is based entirely on stimulus-driven saliency signals.

Similar to Theeuwes $(1991,1992)$, Pashler (1988) found that an irrelevant color singleton interfered with a task involving the localization of a form singleton when the precise target form was unspecified. This suggests that singletons provide a powerful stimulus-driven interference effect. Contrary to Theeuwes's (1992) findings, however, Pashler's data suggest that when the precise form of the target was known, the effect of irrelevant color singletons was mitigated. Using a texture segregation task he termed cross-dimensional texture segregation, Pashler presented displays of 90 elements in which the target was a single unique element (or singleton) that differed from the background on one dimension (e.g., form). Pashler found that random or systematic variation on an irrelevant dimension (e.g., color) did not affect performance on a target localization task. For example, subjects were not significantly slower to localize a target when the elements in the display were colored to produce vertical or horizontal stripes or a checkerboard pattern than when the color of the items in the display was homogenous. Irrelevant color singletons (single elements of unique color), however. 
did interfere with the localization of form singleton targets. In one experiment (Experiment 7), he found that the number of singletons present and their precise location (randomly placed on each trial or fixed across trials) did not appear to influence this distraction effect. (Note that singleton is used here to refer to a single element that differs from all the elements immediately adjacent to it. For this reason, there is no contradiction in saying that multiple singletons were present in the display.) Pashler suggested that localizing such targets is stimulus driven and that perhaps the early segmentation of the display occurs on the basis of the detection of coarse "differences" in such a way that the segregation of a potential target resulting from a difference in color is equivalent to the segregation of one resulting from a local form difference.

\section{Focus of the Present Research}

The main focus of the present research is to examine the role of stimulus-driven (or bottom-up) and goal-directed (or top-down) control of attentional processing. The experiments presented here will examine the factors involved when subjects search through a dense texture display in which form and color differences are presented. Previous research has shown that goal-directed information concerning the specific feature identity of the target (e.g., Pashler, 1988), or the target-defining dimension (e.g., Folk et al., 1992) can reduce or eliminate the effect of distractors defined by the irrelevant dimension. In the present experiments, a different kind of goal-directed information is provided to test whether it, too, can reduce or eliminate the interference from irrelevant distractors. This goal-directed information concerns the overall shape of a target region defined by a discontinuity in a texture as opposed to the precise feature value of the individual texture elements that make up the target discontinuity. There is some evidence that subjects can selectively attend to a particular stimulus dimension (e.g., Folk et al., 1992), although this is not universally accepted (e.g., Theeuwes, 1994; Yantis, 1993b). Can subjects selectively attend to a particular overall shape defined by a particular stimulus dimension? This research should provide additional evidence regarding whether or not (and under what circumstances) this type of goal-directed information can be used to guide the selection of potential targets.

In many studies designed to assess the functioning of attention, singletons, especially singleton distractors, have played a major role. Is this special role warranted? Pashler (1988) suggested that singletons are special because variation in an irrelevant dimension produced a large interference effect only when this variation took the form of singletons. Recall, however, that Pashler used only singleton targets, which might explain why only singleton distractors interfered. Is this distraction effect due to the fact that singletons are preferentially processed, or is this result due to the fact that subjects were looking for targets defined as singletons (even though they were defined on a different dimension than the distractors), and the targets and distractors shared the same overall shape?
The present experiments sought to test Pashler's notions about the specialness of singletons as well.

\section{EXPERIMENT 1}

The first experiment was designed to test whether subjects could use a novel source of goal-directed information to reduce the interfering effects of irrelevant stimulusdriven information in a task and display similar to Pashler's (1988). In addition, this experiment enables a critical test of whether there is anything special about single unique elements (or singletons) in a texture display.

In the first experiment, each stimulus display consisted of a matrix of 90 elements. A few examples of possible displays are shown in Figure 1. Subjects searched for a target of known overall shape. Targets consisted of a single element or a configuration of three elements that differed from the rest of the elements in the display on the basis of form. Targets were singletons, in the case of the single unique form element, or were composed of three elements arranged as a vertical column or a horizontal row. The subjects' task was to indicate whether the target was presented on the left or the right of the display. A target was presented on every trial. One additional factor was manipulated: the presence or absence of (and if present, the nature of) irrelevant color variation. If color variation was present, one of three different distractor configurations was presented by displaying one or three elements in a different color from the rest of the display. These elements made up distractor singletons, rows, or columns identical in size and overall shape to the three kinds of targets. For example, in Figure 1A there is a column target on the right side and no distractor. Figure $1 \mathrm{~B}$ shows a row target on the left and two singleton distractors. Figure $1 \mathrm{C}$ shows a singleton target and two row distractors. Figure 1D shows a column target and two column distractors. Note that these are just a small sample of all possible display combinations.

If the presence of any distractor created by altering the color of one or a few elements in the display interferes with subjects' localization of a form target, this would suggest that subjects are unable to override the attentional capture of a salient "object." This would be shown by similar interference effects, relative to a control condition with no distractors present, for all distractor types. If, on the other hand, no distractor interferes with target detection, this would suggest that subjects are able to override the effects of irrelevant color variation (i.e., perfect goal-directed guidance using information about the relevant dimension). In this case, there would be no interfering effects: mean RTs in the distractor conditions would not be significantly different from control.

More interestingly, if only distractors of the same overall shape (i.e., "matched distractors"; see Figure 1D) interfere, this would suggest that information about target shape is available so that subjects can compare areas of local feature difference to the relevant target template. This goal-directed source of information is not useful, 
A.

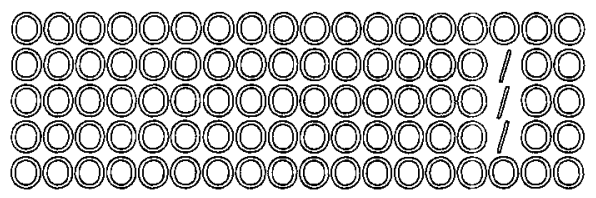

B.

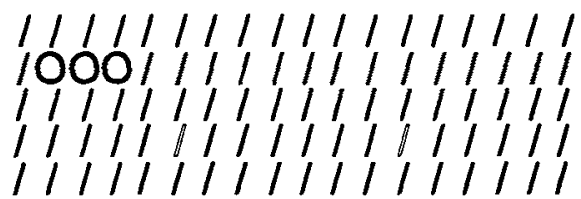

C.

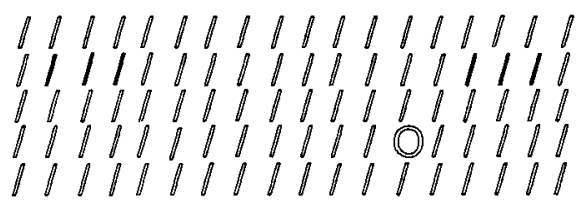

D.

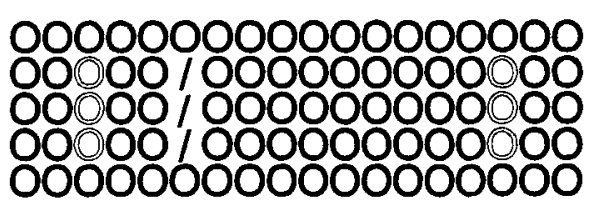

Figure 1. Sample stimulus displays from Experiment 1. (A): an example of a control trial, in which no distractor is presented. The target is made up of three forms $(/)$ presented in white among white $O$ 's. (B): a row target made up of black $O$ 's and two singleton distractors (white /'s). (C): a singleton target (white $O$ ) among white /'s. Distractors in this display are black rows made up of /'s. (D): a vertical column target made up of black /'s and two vertical distractors made up of white $O$ 's. $B$ and $C$ are examples of nonmatching distractors, whereas $D$ is an example of a matching distractor. Actual stimuli were red and green.

however, if the distractors are of the same overall shape as the target. This conclusion would be supported by elevated RTs when the distractor configuration is identical to the target configuration, but RTs to other distractors should not be significantly different from the control condition. Models that posit control or guidance of attention driven solely by stimulus-driven processes would not predict any goal-directed guidance based on overall target shape. These models would predict roughly equal interfering effects from all three distractor configurations. The outcomes discussed here assume equal salience of the three distractor types. More complex predictions are possible if this assumption is relaxed. We return to this point later.

Finally, if there is something uniquely salient about a single discrepant item, then we would expect greater in- terference for all target types when singleton distractors are presented.

\section{Method}

Subjects. Subjects were 34 undergraduate students at Johns Hopkins University who participated in order to partially fulfill a research requirement for an introduciory psychology course. All subjects reported normal or corrected-to-normal visual acuity and normal color vision.

Apparatus and Stimuli. The stimuli were generated by an IBMcompatible computer and displayed on a 15 -in. color VGA monitor. Each display consisted of 90 colored elements arranged in five rows of 18 elements each. Viewing distance was not fixed, but most subjects viewed the display from a distance of approximately $35 \mathrm{~cm}$. The overall dimensions of the display were $8.2 \mathrm{~cm}$ in width $x$ $2.5 \mathrm{~cm}$ in height. Individual elements measured $0.3 \mathrm{~cm}$ in width $X$ $0.4 \mathrm{~cm}$ in height. Each element was an "O" or a "." On each trial the identity of the element used to define the target was chosen from among these two elements.

On one half of the trials, the target was presented on the right side of the display; on the other half of the trials, the target was presented on the left side of the display. Four experimental conditions were created by manipulating the color of the elements in the display. In the control condition, all of the elements appeared in the same color (red or green, standard VGA colors). In the three distractor conditions, distractors were created by drawing a subset of elements in the opposite color from the rest of the array. Each distractor could be a single element, three elements arranged in a vertical column, or three elements arranged in a horizontal row. In order to reduce the possibility that subjects might make the opposite localization response on the basis of the detection of a distractor rather than on the basis of the detection of a target, two identical distractors were placed symmetrically about the middle of the display when distractors were presented. There were two potential locations for each type of distractor or target on each side of the display. These potential locations were constrained so that the target and distractors never appeared in any of the central four columns of the display or in any location around the border of the array. Additionally, no target could appear adjacent to a distractor.

Procedure. Each subject participated in a single experimental session. Subjects were given written instructions on the computer monitor. The instructions informed subjects that a target defined by an item or items that differed from the background in form would be presented on one side of the display and that their task was to respond with a buttonpress to indicate the side of the display on which the target was presented. Responses were made using a custom twobutton response box connected to the computer through the game port. Subjects pressed the left button with a finger of their left hand to indicate a target on the left side of the display, and the right button with a finger of their right hand to indicate a target on the right side. Subjects were instructed to respond as quickly as possible while still maintaining accuracy. The instructions also informed subjects of the irrelevance of the presence of any color variation. Each session consisted of three practice blocks of 30 trials each (one block with each type of target), followed by three experimental blocks of 192 trials each. Each experimental block consisted of 48 trials in each of the four experimental conditions. Within these 48 trials the identity of the element that constituted the target, the color of the distractor, and the location of the distractor were randomly varied so that each possible combination appeared. Before each practice and experimental block, the subject was informed of the overall target shape for that block. The order of presentation of each target shape was counterbalanced across subjects. Each experimental block began with 10 practice trials. Throughout the experiment the computer gave feedback to the subjects via tones. Data were not collected for any practice trials. 
Table 1

Mean Reaction Times (in Milliseconds) in Experiment 1

\begin{tabular}{lcccc}
\hline & \multicolumn{4}{c}{ Distractor Condition } \\
\cline { 2 - 5 } $\begin{array}{c}\text { Target } \\
\text { Condition }\end{array}$ & $\begin{array}{c}\text { Control } \\
\text { (No Distractor) }\end{array}$ & Singleton & Row & Column \\
\hline Singleton & 464 & 505 & 505 & 502 \\
Row & 447 & 471 & $498^{*}$ & 479 \\
Column & 441 & 468 & 476 & $494^{*}$ \\
\hline
\end{tabular}

Note-Means in all distractor conditions are significantly different from the control condition of the same row. *A mean within a row that differs at $p<.001$ by planned comparison from the other distractor conditions.

Subjects initiated a trial by pressing a button on the button box. Each trial began with the presentation of a central fixation cross for $1,200 \mathrm{msec}$. The fixation cross disappeared and $50 \mathrm{msec}$ later the display appeared for $150 \mathrm{msec}$. Subjects pressed one of two buttons to indicate the side of the display on which a target was presented. If a response was incorrect, a $600-\mathrm{Hz}$ tone was presented for $750 \mathrm{msec}$ after the response. Subjects were allowed to rest several times during each block and were required to take a break between blocks.

\section{Results}

Four subjects were removed; 2 were removed because their overall error rates exceeded a preset criterion of $10 \%$, and 2 because of technical problems that occurred during their session. For the remaining 30 subjects, the group mean correct RTs for all target conditions (singleton, row, and column) and each distractor condition are shown in Table 1. RTs greater than $1,200 \mathrm{msec}$ or less than $200 \mathrm{msec}$ were discarded from the analysis, resulting in a loss of less than $1 \%$ of trials.

A repeated measures analysis of variance (ANOVA) (target condition $\times$ distractor condition) revealed a significant main effect of distractor condition $[F(3,87)=103.76$, $p<.001]$ and of target condition $[F(2,58)=8.84, p<$ $.001]$ as well as a target condition $\times$ distractor condition interaction $[F(6,174)=13.99, p<.001]$. For each target shape, mean RT was significantly greater in each of the distractor conditions than in the control condition. Overall RT was slower for singleton targets than for row and column targets.

In order to assess the specific hypothesis that matched distractors would interfere more than nonmatched distractors, we used contrast analysis to compare RT in the matched distractor condition with the average RT in the two nonmatched distractor conditions combined. Planned comparisons revealed significantly longer RTs for distractors that matched the overall shape of the target than for those that did not match the overall target in the row target $[F(1,29)=39.57, p<.001]$ and column target $[F(1,29)=47.11, p<.001]$ conditions. For the case of singleton targets, singleton distractors did not cause significantly greater interference than row or column distractors $[F(1,29)<1]$.

The same overall ANOVA on error rates revealed a significant effect of distractor condition $[F(3,87)=3.70$, $p<.05]$. Subjects made more errors when column distractors were presented. These data are shown in Table 2.
This pattern does not show evidence of a speed-accuracy tradeoff or any reason to doubt the results of the RT data. The overall error rate was $4.1 \%$ and individual subject error rates ranged from $1 \%$ to $9 \%$.

\section{Discussion}

The results of Experiment 1 show interference from all distractors, even though these distractors were known to be irrelevant to the task. Given the results of Pashler (1988) and Theeuwes $(1991,1992)$, this first result is not surprising. What is more interesting is the second resultthat distractors that matched the overall shape of the target showed significantly greater interference when subjects localized row and column targets. Mismatched distractors also interfered, suggesting that they were salient, yet they did not cause as great an interfering effect as did the matching distractors. Because target shape was blocked, subjects knew in advance of the trial what the target shape would be. This result therefore suggests that this information provides goal-directed guidance that can reduce interfering effects of even salient distractors on the basis of overall shape and argues against a purely stimulus-driven account of these distraction effects. This is problematic for Theeuwes, who has argued (Theeuwes, 1991, 1992, 1994) that the saliency of an item (i.e., the strength of its stimulus-driven signal) is the only information that the attentional system can use for guidance. Similar accounts have been proposed by Koch and Ullman (1985) and Sagi and Julesz (1985a, 1985b). Note that salience may have an effect on the strength of the stimulus-driven signal and thus on its ability to interfere. Row and column distractors may have interfered with the detection of singleton targets because rows and columns were three times larger than singletons. This is consistent with Theeuwes as well as others (Folk \& Remington, 1998; Folk et al., 1994). Any account of these results based on salience or size alone, however, cannot account for the specific matched interference for rows and columns, respectively. These row and column distractors both featured three unique color elements, which, it could be argued, would provide equal saliency signals, and yet they showed global shapespecific interference effects.

The present results are consistent with those of Pashler (1988), who found that irrelevant singletons interfered with the localization of singleton targets. Recall that Pashler's subjects were always looking for singleton (i.e., singleelement) targets. Although Pashler tested only these singleelement targets (and found significant interfering effects

Table 2

Mean Error Rates (\%) in Experiment 1

\begin{tabular}{lcccc}
\hline & \multicolumn{3}{c}{ Distractor Condition } \\
\cline { 2 - 5 } $\begin{array}{c}\text { Target } \\
\text { Condition }\end{array}$ & $\begin{array}{c}\text { Control } \\
\text { (No Distractor) }\end{array}$ & Singleton & Row & Column \\
\hline Singleton & 3.4 & 4.4 & 5.0 & 5.5 \\
Row & 4.3 & 4.2 & 3.4 & 4.0 \\
Column & 3.4 & 3.5 & 3.4 & 5.3 \\
\hline
\end{tabular}


for singleton distractors), his accounting of his results suggested that singletons are special because they are regions of high dissimilarity. The present experiment yielded no evidence that single elements are special. If the definition of singleton is broadened, however, to include adjacent elements that group into regions of high dissimilarity (i.e., the row and column distractors), the results are consistent with the premise that singletons are special. The present results, though, go beyond Pashler's by showing that regions of high dissimilarity that match the overall shape of the target are even more distracting than regions that do not match, suggesting that not all "singletons" are created equal.

Pashler (1988) suggested that these multielement texture displays are segregated into likely target locations on the basis of differences between elements, and that this stimulus-driven segregation provides information about where there are differences in the field, but not necessarily the features (or dimensions) on which these areas are different. We suggest that in this task, this stimulusdriven activation is combined with goal-directed information that subjects have concerning the overall configuration of the target. Distractors that match the overall target configuration caused greater interference because the goal-directed information about shape was not useful in eliminating these distractors from further processing. If a distractor cannot be eliminated on the basis of overall shape, further processing is required to determine which "difference" signal corresponds to the target. If all distractors had provided equal interference, we would conclude that subjects cannot use information about the overall shape of the target to reduce the interfering effects of these distractors. The fact that irrelevant color distractors caused less interference when they did not match the overall target configuration suggests that these distractors were eliminated from further processing. Note that some interference occurred despite the fact that the dimension on which the distractors were defined was known to be irrelevant, showing that although information about the overall shape of the target was beneficial to subjects, knowledge about the relevant dimension was not sufficient to eliminate all interference from these salient distractors.

\section{EXPERIMENT 2}

The results of Experiment 1 show that distractors that match the overall target configuration interfere with the localization of targets to a greater extent than distractors that do not match the target. This suggests that goaldirected information can be used to mitigate the interfering effects of irrelevant but salient distractors. One possible concern that might arise is that the pattern of results observed in Experiment 1 might be due to the specific assignment of form as the target-defining dimension and color as the irrelevant dimension. Callaghan, Lasaga, and Garner (1986) found asymmetric performance in a similar texture segregation task when they varied color (hue) and orientation. Their results suggested that irrelevant variation in hue interfered more with detection of a target defined by orientation than irrelevant variation in orientation interfered with targets defined by hue. Similarly, Theeuwes (1991, Experiment 2) found that visual search for a unique form was slowed by the presence of a display element with a unique color, but search for a unique color was not slowed by the presence of a unique form. In order to address this concern, Experiment 2 was designed exactly like Experiment 1 except that the target and distractor dimensions of Experiment 1 were reversed.

In Experiment 1, distractors were defined by elements that differed in color from the background and targets were defined by elements that differed in form. In this experiment, targets were defined by color differences and distractors by form differences. This experiment sought to examine whether the color differences present in these displays are more salient than the form differences present and if this might account for the results of Experiment 1 . If the same pattern of results is found in this experiment with form distractors and color targets, we could conclude that the effect observed in Experiment 1 was not due solely to the assignment of relevant and irrelevant dimensions.

\section{Method}

Subjects were 24 undergraduate students at Johns Hopkins University who participated in order to partially fulfill a course requirement. The apparatus and stimuli were identical to those of Experiment 1 except that in this experiment the target was defined by elements presented in a color that differed from the rest of the items in the display (e.g., red elements among green elements), arranged in the same target and distractor configurations as in Experiment 1. This time the distractors consisted of elements that differed from the background elements on the basis of form (e.g., O's among/'s). A few examples of possible displays are shown in Figure 2. As in Experiment 1, subjects were to localize the target on the left or the right side of the display under the same four distractor conditions. The procedure was the same as in Experiment 1.

\section{Results}

The results are shown in Tables 3 and 4 . Table 3 shows mean correct RT as a function of target and distractor conditions. Table 4 shows mean error rate as a function of target and distractor conditions. RTs greater than $1,200 \mathrm{msec}$ or less than $200 \mathrm{msec}$ were discarded, resulting in the loss of less than $1 \%$ of trials.

A repeated measures ANOVA (target condition $\times$ distractor condition) run on the mean correct $\mathrm{RT}$ data showed a significant effect of target condition $[F(2,46)=6.77$, $p<.01]$ and of distractor condition $[F(3,69)=5.23, p<$ $.01]$. The interaction was not significant $(p>.10)$. The same planned comparisons as in Experiment 1 again showed that subjects took longer to respond when distractors matched the overall shape of the target for row $[F(1,23)=6.92, p<.05]$ and column $[F(1,23)=7.65$, $p<.05]$ targets. Again, this was not true for singleton targets $[F(1,23)<1]$. Although the magnitude of this interfirence effect was not as great as in Experiment 1, this was a consistent pattern, with 18 of 24 subjects showing 
A.

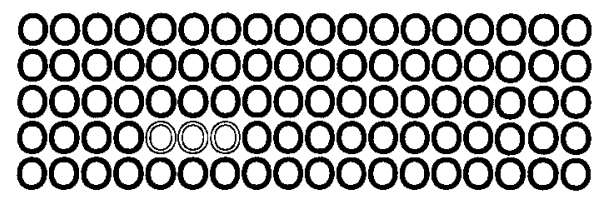

B.

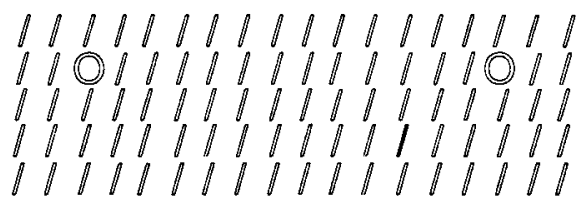

C.

111111111111111111 11111111111111811 1111111111111111 111100011110001111 11111111111111111

D.

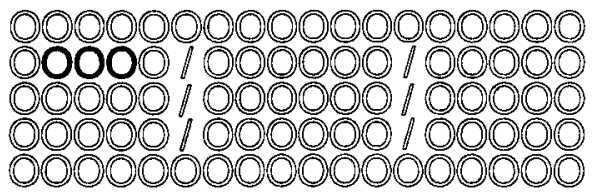

Figure 2. Sample stimulus displays from Experiment 2. (A): an example of a control trial, in which the target is a color row target made up of white $O$ 's among black $O$ 's. (B): a color singleton target (black /) and two singleton distractors (white $O$ 's). (C): a column target made up of white /'s among black /'s and two row distractors made up of black $O$ 's. (D): a row target made up of black O's and two column distractors made up of white /'s. $C$ and $D$ are examples of nonmatching distractors, whereas $B$ is an example of a matching distractor.

slower RTs for matching distractors than for nonmatching distractors. Subjects responded significantly more rapidly to column targets than to row or singleton ${ }^{1}$ targets. Overall, subjects were also significantly faster to respond in the control condition $(M=389 \mathrm{msec})$ than in either the row $(M=395 \mathrm{msec})$ or the column $(M=397 \mathrm{msec})$ distractor condition $(p<.01$ for row distractors, $p<.05$ for columns, by Tukey's HSD). The same overall ANOVA run on the error rates showed no significant effects. Overall error rate was $2.8 \%$ and individual subject error rates ranged from $0 \%$ to $9 \%$.

\section{Discussion}

Two main points can be made. Although the interference effects observed in this experiment were not as great as those observed in Experiment 1, the basic finding that matched distractors interfered more than non- matched distractors remains. This therefore argues against any account of the first experiment based on the specific assignments of target and distractor dimensions. Second, relative saliency of the targets and distractors is important. Comparing the results of this experiment with those of Experiment 1 suggests that form distractors did not interfere with color targets to the same extent that color distractors interfered with form targets in Experiment 1 . Notice, for example, the lack of a significant interference effect between control trials in which no distractor was present and trials in which a distractor was present. Subjectively, the color differences in this experiment were much more salient than the form differences. Thus, this experiment suggests that the salience of the irrelevant item has a profound effect on the strength of the stimulus-driven interference, consistent with the claims of Theeuwes (1991). Folk and Remington (1998) have also recently presented such an account and evidence from their modified spatial cuing task to support such a claim. The asymmetry between color and form is consistent with the findings from other studies using texture segregation (e.g., Callaghan, 1989; Callaghan et al., 1986) and visual search (e.g., Theeuwes, 1991, 1992). These findings have been taken as evidence that the relative discriminability of differences is critical in perception (see also Garner, 1983; Gottwald \& Garner, 1972).

\section{EXPERIMENT 3}

The results of Experiments 1 and 2 suggest that if subjects are told the overall configuration of the target, the degree to which the distractor will interfere is determined by whether or not an irrelevant distractor matches the target. If the irrelevant distractor matches the overall configuration, RT to localize the target is increased relative to nonmatching distractors. We hypothesized that the results of Experiments 1 and 2 were due to goal-directed guidance based on the overall shape of the target. In order to test this hypothesis, it is necessary to examine a condition in which subjects are not informed of what target configuration to expect on each trial. According to our hypothesis, if subjects are not provided specific goaldirected information about targets, roughly equivalent interference effects from all types of distractors (with all types of targets) should obtain. In this experiment, target type was mixed rather than blocked, making the overall target shape unpredictable on each trial.

Table 3

Mean Reaction Times (in Milliseconds) in Experiment 2

\begin{tabular}{lcccc}
\hline & \multicolumn{4}{c}{ Distractor Condition } \\
\cline { 2 - 5 } $\begin{array}{c}\text { Target } \\
\text { Condition }\end{array}$ & $\begin{array}{c}\text { Control } \\
\text { (No Distractor) }\end{array}$ & Singleton & Row & Column \\
\hline Singleton & 396 & 402 & 401 & 403 \\
Row & 393 & 394 & $402^{*}$ & 398 \\
Column & 380 & 384 & 382 & $390^{*}$ \\
\hline
\end{tabular}

*A mean within a row that differs at $p<.05$ from the other distractor conditions. 
Table 4

Mean Error Rates (\%) in Experiment 2

\begin{tabular}{|c|c|c|c|c|}
\hline \multirow[b]{2}{*}{$\begin{array}{c}\text { Target } \\
\text { Condition }\end{array}$} & \multicolumn{4}{|c|}{ Distractor Condition } \\
\hline & $\begin{array}{c}\text { Control } \\
\text { (No Distractor) }\end{array}$ & Singleton & Row & Column \\
\hline Singleton & 2.3 & 2.2 & 2.8 & 2.0 \\
\hline Row & 2.3 & 2.9 & 3.1 & 2.7 \\
\hline Column & 4.0 & 3.5 & 3.2 & 3.1 \\
\hline
\end{tabular}

If on every trial subjects are required to search for a target in any of the three possible target configurations, they should be unable to reduce interference from distractors that do not match the target, and, therefore, all distractors should provide roughly equal interference.

\section{Method}

Subjects were 29 undergraduate students at Johns Hopkins University who participated in order to partially fulfill a course requirement. The apparatus and stimuli were identical to those of Experiment 1 . The procedure was identical to that of Experiment 1 except that in this experiment the overall shape of the target was not held constant for a block of trials, but was varied from trial to trial. Subjects were told that on each trial the target could be a single unique form element, a vertical column of three unique form elements, or a horizontal row of three unique form elements.

\section{Results}

Five subjects were removed due to overall error rates above $10 \%$. For the remaining 24 subjects the group mean RT as a function of target and distractor conditions is shown in Table 5. Reaction times greater than $1,200 \mathrm{msec}$ or less than $200 \mathrm{msec}$ were discarded, resulting in a loss of less than $1 \%$ of the trials.

The same repeated measures ANOVA as in Experiment 1 was run on these data. The RT analysis revealed a significant effect of target condition $[F(2,46)=44.39$, $p<.001]$ and of distractor condition $[F(3,69)=109.44$, $p<.001]$ and a significant target condition $\times$ distractor condition interaction $[F(6,138)=2.71, p<.05]$. Subjects were faster to respond in the control condition than in any of the distractor conditions. As in Experiment 1, subjects were slower to respond to singleton targets. Planned comparisons revealed a significant effect of singleton distractors on singleton targets $[F(1,23)=10.86, p<.01]$; however, this effect was in the opposite direction from that predicted by the matching hypothesis. Subjects were faster to respond to singleton targets when presented with singleton distractors than when presented with row or column distractors.

The error rate data revealed a significant effect of distractor condition $[F(3,69)=3.53, p<.05]$. Subjects made more errors when singleton distractors were presented. These data are shown in Table 6 . The overall error rate was $3.9 \%$ and individual subject error rates ranged from $0.8 \%$ to $9 \%$.

In order to test the hypothesis that subjects in Experiment 1 were able to reduce interference from nonmatching distractors whereas subjects in this experiment were not, we ran an ANOVA on the RT data of Experiments 1 and 3 together. This analysis compared the mean RT in the conditions in which the distractors matched the targets with the mean RT in those in which they did not, across the two experiments. For each subject we computed the mean RT for all conditions in which the distractors matched the targets, averaged across overall target shape (i.e., singleton target and singleton distractors, row target and row distractors, and column target and column distractors combined) and the mean RT for all nonmatching distractor conditions combined, also averaged across overall target shape. This was done separately for each experiment. In Experiment 1, the mean overall RT was $499 \mathrm{msec}$ for matching distractor conditions and $484 \mathrm{msec}$ for nonmatching distractors. For Experiment 3, the mean overall RT was $495 \mathrm{msec}$ for matching distractors and $498 \mathrm{msec}$ for nonmatching distractors. We then subjected these data to a 2 (experiment) $\times 2$ (distractor condition) mixed ANOVA. The analysis revealed a significant effect of distractor condition $[F(1,52)=19.60, p<.001]$ and an interaction of experiment and distractor condition $[F(1,52)=34.74, p<.001]$. An analysis of the interaction using planned comparisons revealed that the effect of distractor condition was significant in Experiment 1 $[F(1,52)=59.92, p<.001]$ but was not significant in Experiment $3[F(1,52)<1$, n.s.]. This analysis shows that subjects were significantly slower in responding to targets in the presence of matching distractors than in the presence of nonmatching distractors in Experiment 1 , but there was no difference between matching and nonmatching distractors in Experiment 3. The preceding analysis does not take into account the mean difference in control RTs of Experiments 1 and 3. To do so, each subject's control RT was subtracted from his/her mean RT in the matching distractor and nonmatching distractor conditions. These difference scores were then entered into an analysis identical to the one described just above. The results were essentially the same.

\section{Discussion}

The results of Experiment 3 suggest that all distractors showed roughly equivalent amounts of interference with the localization of all targets. This interference occurred even though all distractors were known to be irrelevant to the task. In contrast to the results of Experiments 1 and 2, in which distractors that matched the overall shape of the target (i.e., the rows and columns) showed

Table 5

Mean Reaction Times (in Milliseconds) in Experiment 3

\begin{tabular}{lcccc}
\hline & \multicolumn{3}{c}{ Distractor Condition } \\
\cline { 2 - 5 } $\begin{array}{c}\text { Target } \\
\text { Condition }\end{array}$ & $\begin{array}{c}\text { Control } \\
\text { (No Distractor) }\end{array}$ & Singleton & Row & Column \\
\hline Singleton & 454 & $502 *$ & 517 & 511 \\
Row & 441 & 492 & 492 & 483 \\
Column & 442 & 492 & 491 & 492 \\
\hline
\end{tabular}

Note-Means in all distractor conditions are significantly different from the control condition of the same row. *A mean within a row that differs at $p<.05$ by planned comparison from the other distractor conditions. 
Table 6

Mean Error Rates (\%) in Experiment 3

\begin{tabular}{lcccc}
\hline & \multicolumn{4}{c}{ Distractor Condition } \\
\cline { 2 - 5 } $\begin{array}{c}\text { Target } \\
\text { Condition }\end{array}$ & $\begin{array}{c}\text { Control } \\
\text { (No Distractor) }\end{array}$ & Singleton & Row & Column \\
\hline Singleton & 2.4 & 4.7 & 4.5 & 3.4 \\
Row & 4.0 & 4.8 & 4.2 & 4.5 \\
Column & 2.8 & 3.9 & 3.9 & 3.7 \\
\hline
\end{tabular}

greater interference, in this experiment no such "match effect" was found. These results are consistent with the hypothesis that if subjects do not have a target template based on overall shape, they are about equally susceptible to the interfering effects of all types of irrelevant distractors. Comparison of the results of this experiment with those of Experiment 1, in which the distractors were as salient, indicates that subjects in this experiment were more susceptible to interference from nonmatching distractors than were subjects in Experiment 1. The interfering effect of these nonmatching distractors in this experiment is roughly equal to the amount of interference from matching distractors obtained in Experiment 1. This result supports the claim that subjects in Experiment 1 were able to use goal-directed information about the overall shape of the target to reduce interference from nonmatching distractors.

\section{GENERAL DISCUSSION}

The present experiments were designed to assess the role of stimulus-driven and goal-directed sources of control of visual attention. Many theoretical accounts of visual information processing have suggested that these two different sources of information are important for the control or guidance of selective attention. Some accounts emphasize the importance of goal-directed sources of information (e.g., Bundesen, 1990; Hoffman, 1979), whereas other accounts emphasize the stimulus-driven properties of the stimulus to the exclusion of goal-directed influences (e.g., Koch \& Ullman, 1985; Sagi \& Julesz, 1985a, 1985b). Yet other accounts have acknowledged significant contributions of both types (e.g., Treisman \& Sato, 1990; Wolfe, 1994).

Theeuwes $(1991,1992)$ has argued that selection is never goal directed, basing this conclusion on his finding that in a visual search task in which the target is defined along one dimension, subjects cannot avoid the interfering effects of a salient singleton item presented in the display, even when they know it is irrelevant. This effect occurs even when subjects are fully informed of what target to expect. Pashler (1988) also found similar evidence for the importance of (i.e., attentional priority given to) irrelevant singletons using a texture segregation task. Unlike Theeuwes, however, Pashler found that if subjects were given goal-directed information concerning the target, they could reduce the interfering effects of salient singleton distractors.
The experiments reported here provide additional evidence for the joint operation of both stimulus-driven and goal-directed guidance of attentional processing in a task requiring the selection of a relevant target from a display that also contained irrelevant information. Unlike previous studies, which have examined the interaction of these two sources of attentional control by providing information about relevant stimulus features of individual elements such as shape, color, motion, or abrupt onset, the present study has shown the importance of goal-directed guidance to a different level of representation, that of the overall shape of a group of elements. In Experiment 1, targets were defined by elements in the form dimension; variation in the color dimension was irrelevant. In Experiment 2 , the relevant and irrelevant dimensions were reversed. In both of these experiments the overall shape of the target was known. In these experiments, distractors that did not match the overall shape of the target caused less interference than distractors that matched the target's shape. In Experiment 3, targets were again defined by differences in form and distractors by differences in color; however, subjects were not informed of the overall shape of the target. In this experiment all distractors caused roughly equal interference. A comparison between Experiments 1 and 3 suggests that goal-directed information concerning the overall shape of the target enabled subjects to reduce the amount of interference from distractors that did not match the overall target shape. This finding argues against purely stimulus-driven accounts of attentional guidance, as proposed by Theeuwes (1991, 1992) and others (e.g., Koch \& Ullman, 1985; Sagi \& Julesz, 1985a, 1985b). It is obvious, however, that stimulusdriven information does play an important role, as shown by the difference between any distractor condition and the control condition in Experiments 1 and 3.

As mentioned previously, other studies have provided evidence that the salience of a stimulus, whether target or distractor, may play a role in determining the degree of stimulus-driven capture (Theeuwes, 1991, 1992, 1994) and the amount of goal-directed attentional control possible (Folk \& Remington, 1998; Folk et al,, 1994). We interpret our results to be consistent with this view based on three findings. First, the fact that the localization of singleton targets in Experiment 1 was disrupted by column and row distractors as much as by singletons suggests that the size of these larger distractors made them more difficult to ignore. (Remember, though, that salience alone cannot account for all of the interference effects because matching distractors interfered more with row and column targets than nonmatching distractors of the same size.) Second, in Experiment 2 form distractors composed of elements that differed from the background did not interfere with color-defined targets to the same extent that the color-defined distractors of Experiment 1 interfered with form targets, suggesting that color differences that were subjectively greater made odd-colored items (distractors in Experiment 1 and targets in Experiment 2) more salient. Finally, even nonmatching distrac- 
tors in Experiment 1 (and all distractors in Experiment 3) caused significant interference compared with the control condition, in which no distractor was presented, suggesting that any salient difference provides a stimulusdriven signal that cannot be entirely ignored.

The results of these experiments are also relevant to the contingent involuntary orienting hypothesis of Folk et al. (1992). Folk et al. (1992) proposed that under conditions of spatial uncertainty, irrelevant stimulus events capture attention only when the event shares a feature property that is critical to performing the task at hand. In our displays this account would suggest that attention is captured by the discontinuities that correspond to distractors, according to how well they match the attentional target of the observer. When the observer does not know the shape of the target (as in Experiment 3), this feature is not available to guide attention, and all discontinuities (regardless of their overall shape) capture attention, but when the observer knows the shape of the target, distractors that do not match the target cause less interference (as in Experiments 1 and 2). Consistent with the findings of Folk et al. (1992), this suggests that the attentional control system may not be able to distinguish between feature dimensions. In other words, it cannot tell a color singleton (column or row) from a form singleton (column or row). If it could, there would be no interference from a dimension known to be irrelevant. (See Folk \& Remington, 1998, however, for evidence that subjects can set for a particular feature value.)

The fact that greater interference is found for matching distractors (i.e., reduced interference is found for nonmatching distractors) when overall target shape is known suggests that this mechanism can give a coarse representation of the overall shape (e.g., it can tell the difference between a singleton and a column). This demonstration of a new source of goal-directed information suggests additional flexibility in the mode of control of selective attention and may represent an additional attentional control setting, or perhaps a different class of settings that can be used. The finding that all distractors interfered is consistent with Pashler's (1988) suggestion that regions of high dissimilarity are special, regardless of whether the dimension that defines them is relevant. This would account for why Pashler found that variation in an irrelevant dimension did not interfere unless the variation created singletons, because his subjects were always looking for singleton targets. This is in fact the interpretation of Pashler's results proposed by Folk et al. (1992).

We have interpreted our results as providing evidence for goal-directed attentional selection. It has been suggested by Theeuwes (1994) that the greater interference of matching distractors observed in the experiments of Folk et al. (1992) might be due to an inability to disengage attention rapidly from distractors that share a common feature with the intended target, while disengagement from distractors that do not share this feature is more rapid. Unlike Folk et al.'s account, which is based on goal-directed guidance of attentional selection, Theeuwes's account stresses an effect of goal-directed information on processes occurring after selection. The same argument could be used to account for our results. Although this possibility cannot be ruled out on the basis of the data presented here, Folk and Remington (1998, Experiment 4) did directly test this idea using their modified spatial cuing task and found no evidence to support Theeuwes's (1992) disengagement account. Nevertheless, it remains to be determined whether the influence of goal-directed information observed here affects attention at the level of selection.

Another question that arises concerns whether the results of the experiments presented here can be accounted for by response competition. We have proposed that the influence of goal-directed information occurs at the level of perceptual selection. In contrast, a response competition account suggests that response interference occurs when distractors that are similar to the target are on opposite sides of the display, activating the competing response. When the target definition is narrowed by providing subjects with goal-directed information, the range of potentially competing stimuli is narrowed as well, thus reducing the interference. Previous research in our laboratory suggests that whether all distractors appear on the same or opposite side of the display has no effect on the amount of interference by matching or mismatched distractors, indicating that the results are not due to response competition (Ghirardelli, 1996).

Further evidence against a response competition account is provided by Pashler (1988). He argued that the results of his Experiment 1, which used an accuracy measure rather than RT, suggest that the interference he observed was not a response competition effect because accuracy in masked displays is most likely due to the perceptual component of the task and not the response selection component. In such a nonspeeded task, subjects presumably allow enough time for the response selection stage to proceed to asymptotic accuracy levels. While all of the experiments reported here used a speeded response, our claim that this is not a response compatibility effect is bolstered by the fact that our results are consistent with what Pashler found using both speeded and nonspeeded tasks. Additional experiments are planned to address this question further.

\section{Further Implications}

Singleton search. Bacon and Egeth (1994) have previously argued against Theeuwes's $(1991,1992)$ accounts of his findings, using a slight variant of his task. Like Theeuwes, they provided subjects with goal-directed information about the relevant target dimension along with salient but task-irrelevant information in the form of a singleton defined on the irrelevant dimension. They showed that when goal-directed and stimulus-driven information conflict, it is possible to override stimulus-driven capture. They accounted for Theeuwes's results by proposing that subjects in his tasks (and their Experiment 1) adopted a strategy they called singleton detection mode. Observers in singleton detection mode rely on identify- 
ing elements that differ from their background, but not by attending to any particular feature (as in feature search mode). (Bacon and Egeth [1994] also suggested that in displays with more than one "odd item," a more general term, discrepancy detection mode, applies to the same idea.) When this strategy was made unattractive to subjects (e.g., by increasing the number of discrepant shapes in each display), they adopted a feature search mode, which uses goal-directed information about the relevant target feature to eliminate the interfering effects of irrelevant stimuli. Note that although this explanation can account for Theeuwes's results, the adoption of neither singleton detection mode nor discrepancy detection mode would help subjects in the experiments reported in this study. In these experiments, detecting a discrepancy would not have helped subjects find the target except on control trials because the distractors were also discrepancies in the texture. Because control trials occurred relatively infrequently in the experiments reported here, it is assumed that subjects did not attempt this strategy, and therefore the effects observed cannot be accounted for by the use of such strategies.

Subjects in Pashler's (1988) study may also have adopted a singleton detection mode. In that study, targets were defined as singletons, and thus singleton detection mode represented a strategy using both goal-directed and stimulus-driven sources of information. This may explain why Pashler found that irrelevant singletons were particularly disruptive. The present study has shown that although regions of high dissimilarity may have high priority in visual information processing (i.e., may be special), single featural elements (feature singletons) do not appear to automatically capture attention in a purely stimulus-driven manner (i.e., they are not necessarily special). This finding was anticipated by Pashler and is widely supported by recent experiments (e.g., Folk \& Annett, 1994; Folk et al., 1992; Hillstrom \& Yantis, 1994; Jonides \& Yantis, 1988; Todd \& Kramer, 1994; Yantis \& Egeth, 1994; for a more detailed discussion of this topic, see Egeth \& Yantis, 1997; Folk \& Remington, 1996; Yantis, 1996).

Texture segregation. Of additional theoretical interest, the results of these experiments are consistent with the idea that texture segregation is an early (perhaps preattentive) process that serves to organize a scene into perceptual units such as figure and ground. Such a model has been proposed by Grossberg, Mingolla, and Ross (1994), and may well be consistent with Neisser's (1967) original characterization of preattentive processing (see Bravo \& Blake, 1990). This early processing is capable of representing more than simple features in order to provide a more detailed representation for attentive processing. The experiments reported here have shown that variation on a dimension that is known to be irrelevant can interfere with this segregation process, suggesting that this process proceeds automatically across all dimensions.

Furthermore, unlike earlier characterizations of preattentive processing of a limited set of simple features, more recent studies have demonstrated that early preattentive processing can incorporate such complex features as groups of elements (Bravo \& Blake, 1990), surface colors and orientation of perceived 3-D objects (Enns \& Rensink, 1990a, 1990b), and 3-D properties involved in figureground separation (He \& Nakayama, 1992). The present study can be considered to provide additional evidence that early processing goes beyond representing simple features to at least the level of representing coarse shapes.

One question that is of additional interest concerns whether finding the target in these displays can be accomplished in parallel, or whether it must be carried out in a strictly serial manner. Bravo and Blake (1990) showed that groups of elements in a texture display, defined by a shared difference in either luminance or orientation, could be searched in parallel. Each display contained a varied number of groups $(1,3,6$, or 9$)$ that formed rectangles. RT to find the target, defined as a rectangle of a particular orientation, did not increase with increasing display size. Importantly, these displays differed from the displays in this study because on a given trial the groups in Bravo and Blake's displays were formed by variation in only one dimension. The design of these experiments did not permit a critical test of serial or parallel processing of the discontinuities in these displays because when distractors were presented, there were always two of them. We are currently pursuing this question in our laboratory. A preliminary finding that is relevant to this issue can be found in Ghirardelli (1996).

Given that previous studies utilizing the same logic for pitting goal-directed and stimulus-driven processing against each other (e.g., Bacon \& Egeth, 1994; Folk et al., 1992; Folk et al., 1994; Theeuwes, 1991, 1992, 1994) have typically used one distractor, it is worth noting that our displays contained two distractors. As mentioned previously, this was done to ensure that subjects would respond on the basis of locating the target. It is conceivable that this might render stimulus-driven information more influential in our displays than if only a single distractor were present. In other words, it is possible that a single distractor might be easier to ignore than two. Although none of the experiments presented here was designed to test this possibility, a study from our laboratory suggests that varying the number of distractors in these displays between one and three has no overall significant effect on performance (Ghirardelli, 1996, Experiment 4).

Global/local processing. In addition to the implications this research has for theories of attentional control and texture segregation, the present results are also consistent with findings from studies examining global and local processing, specifically the global precedence literature. Global precedence refers to preferential perceptual processing for the more global features of a given object (see, e.g., Navon, 1977; Paquet \& Merikle, 1988). Although the present experiments were not designed to assess global precedence, the results are not inconsistent with previous findings (e.g., Paquet, 1992; Paquet \& Merikle, 1988) showing that attention to global forms is 
not disrupted by irrelevant local items, but that attention to local forms can be interfered with by irrelevant global information. Although global precedence may have had some effect in the present experiments, this should not be surprising since the global shape was a critical piece of the task-relevant information provided to subjects. Unlike the experiments of Paquet (1992; Paquet \& Merikle, 1988), the present experiments did not test global precedence directly (e.g., by pitting global and local information against each other). In the present experiments, targets and distractors were defined by the feature dimension of the local elements as well as by the global shape of the target (when this information was provided). Thus, information at the global and local levels was never inconsistent in the present experiments, but rather was completely redundant. It may be important as well that, unlike in previous studies examining global precedence, the exact feature value of the local elements was irrelevant here, subjects were provided only the feature dimension, and the target was based on global shape. Further research is needed to test the role of global precedence in the effects observed in these experiments.

\section{Conclusions}

Taken together, these results suggest that although some stimulus-driven interference is unavoidable, subjects can use goal-directed information to reduce this interference from salient distractors. The nature of this goal-directed information concerned the coarse overall shape of the target and so provides an instance of a previously unexplored source of such goal-directed guidance. The picture that emerges is one of a visual system capable of combining sources of information, both stimulus-driven and goal-directed, in a dynamic fashion.

\section{REFERENCES}

BacON, W. F., \& EGETH, H. E. (1994). Overriding stimulus-driven attentional capture. Perception \& Psychophysics, 55, 485-496.

BACON, W. F., \& EGETH, H. E. (1997). Goal-directed guidance of attention: Evidence from conjunctive visual search. Journal of Experimental Psychology: Human Perception \& Performance, 23, 948-961.

Bergen, J. R., \& Julesz, B. (1983). Parallel versus serial processing in rapid pattern discrimination. Nature, 303, 696-698.

Bravo, M. J., \& Blake, R. (1990). Preattentive vision and perceptual groups. Perception, 19, 515-522.

Broadbent, D. (1958). Perception and communication. Oxford: Pergamon.

Bundesen, C. (1990). A theory of visual attention. Psychological Review, 97, 523-547.

Callaghan, T. C. (1989). Interference and dominance in texture segregation: Hue, geometric form, and line orientation. Perception \& Psychophysics, 46, 299-311.

Callaghan, T. C., Lasaga, M. I., \& Garner, W. R. (1986). Visual texture segregation based on orientation and hue. Perception \& Psychophysics, 39, 32-38.

Egeth, H. E., \& Yantis, S. (1997). Visual attention: Control, representation, and time course. Annual Review of Psychology, 48, 269-297.

ENNS, J. T., \& RENSINK, R. A. (1990a). Influence of scene-based properties on visual search. Science, 247, 721-723.

Enns, J. T., \& Rensink, R. A. (1990b). Preattentive recovery of threedimensional orientation from line drawings. Psychological Review, 98, 335-351.
Folk, C. L., \& AnNetT, S. (1994). Do locally defined feature discontinuities capture attention? Perception \& Psychophysics, 56, 277-287.

Folk, C. L., \& REMington, R. W. (1996). When knowledge does not help: Limitations on the flexibility of attentional control. In A. F. Kramer, M. G. H. Coles, \& G. D. Logan (Eds.), Converging operations in the study of visual selective attention (pp. 271-296). Washington, DC: American Psychological Association.

Folk, C. L., \& Remington, R. W. (1998). Selectivity in distraction by irrelevant featural singletons: Evidence for two forms of attentional capture. Journal of Experimental Psychology: Human Perception \& Performance, 24, 1-12.

Folk, C. L., Remington, R. W., \& Johnston, J. C. (1992). Involuntary covert orienting is contingent on attentional control. Journal of Experimental Psychology: Human Perception \& Performance, 18, $1030-1044$.

Folk, C. L., Remington, R. W., \& Johnston, J. C. (1993). Contingent attentional capture: A reply to Yantis (1993). Journal of Experimental Psychology: Human Perception \& Performance, 19, 682-685.

Folk, C. L., Remington, R. W., \& Wright, J. H. (1994). The structure of attentional control: Contingent attentional capture by apparent motion, abrupt onset, and color. Journal of Experimental Psychology: Human Perception \& Performance, 20, 317-329.

GARNER, W. R. (1983). Asymmetric interactions of stimulus dimensions in perceptual information processing. In T. J. Tighe \& B. E. Shepp (Eds.), Perception, cognition, and development: Interactional analyses (pp. 1-38). Hillsdale, NJ: Erlbaum.

Ghirardelli, T. G. (1996). Pre-attentive and attentive processing in cross-dimensional texture segregation. Unpublished doctoral dissertation, Johns Hopkins University.

GotTWALD, R. L., \& GARNER, W. R. (1972). Effects of focusing strategy on speeded classification with grouping, filtering, and condensation tasks. Perception \& Psychophysics, 11, 179-182.

Grossberg, S., Mingolla, E., \& Ross, W. D. (1994). A neural theory of attentive visual search: Interactions of boundary, surface, spatial, and object representations. Psychological Review, 101, 470-489.

He, Z. J., \& NaKayama, K. (1992). Surface versus features in visual search. Nature, 359, 231-233.

Hillstrom, A. P., \& YANTis, S. (1994). Visual motion and attentional capture. Perception \& Psychophysics, 55, 399-411.

Hoffman, J. E. (1979). A two-stage model of visual search. Perception \& Psychophysics, 25, 319-327.

JAMES, W. (1890). The principles of psychology. New York: Holt.

JONIDES, J., \& YANTIS, S. (1988). Uniqueness of abrupt visual onset in capturing attention. Perception \& Psychophysics, 43, 346-354.

JOSEPH, J. S., \& OPTICAN, L. M. (1996). Involuntary attentional shifts due to orientation differences. Perception \& Psychophysics, 58, 651-665.

Kaptein, N. A., Theeuwes, J., \& Van der Heidden, A. H. C. (1995). Search for a conjunctively defined target can be selectively limited to a color-defined subset of elements. Journal of Experimental Psychology: Human Perception \& Performance, 21, 1053-1069.

Косн, C., \& Ullman, S. (1985). Shifts in selective visual attention: Towards the underlying neural circuitry. Human Neurobiology, 4, 219-227.

Navon, D. (1977). Forest before trees: The precedence of global features in visual perception. Cognitive Psychology, 9, 353-383.

NeISSER, U. (1967). Cognitive psychology. New York: Appleton-CenturyCrofts.

PAQUET, L. (1992). Global and local processing in nonattended objects: A failure to induce local processing dominance. Journal of Experimental Psychology: Human Perception \& Performance, 18, 512-529.

PAQueT, L., \& MERIKLE, P. M. (1988). Global precedence in attended and nonattended objects. Journal of Experimental Psychology: Human Perception \& Performance, 14, 89-100.

Pashler, H. (1988). Cross-dimensional interaction and texture segregation. Perception \& Psychophysics, 43, 307-318.

Poisson, M. E., \& WILKINSON, F. (1992). Distractor ratio and grouping processes in visual conjunction search. Perception, 21, 21-38.

Posner, M. I., SNyder, C. R. R., \& Davidson, B. J. (1980). Attention and the detection of signals. Journal of Experimental Psychology: General, 109, 160-174.

SAGI, D., \& Julesz, B. (1985a). Detection versus discrimination of visual orientation. Perception, 13, 619-628. 
SAGI, D., \& JulESZ, B. (1985b). "Where" and "what" in vision. Science, 228, 1217-1219.

Theevwes, J. (1991). Cross-dimensional perceptual selectivity. Perception \& Psychophysics, 50, 184-193.

Theeuwes, J. (1992). Perceptual selectivity for color and form. Perception \& Psychophysics, 51, 599-606.

THEEUWES, J. (1994). Stimulus-driven capture and attentional set: Selective search for color and visual abrupt onsets. Journal of Experimental Psychology: Human Perception \& Performance, 20, 799-806.

TodD, S., \& Kramer, A. F. (1994). Attentional misguidance in visual search. Perception \& Psychophysics, 56, 198-210.

Treisman, A., \& Sato, S. (1990). Conjunction search revisited. Journal of Experimental Psychology: Human Perception \& Performance, $16,459-478$

WOLFE, J. M. (1994). Guided search 2.0: A revised model of visual search. Psychonomic Bulletin \& Review, 1, 202-238.

Yantis, S. (1993a). Stimulus-driven attentional capture. Current Directions in Psychological Science, 2, 156-161.

YANTIS, S. (1993b). Stimulus-driven attentional capture and attentional control settings. Journal of Experimental Psychology: Human Perception \& Performance, 19, 676-681.
YANTIS, S. (1996). Attentional capture in vision. In A. F. Kramer, M. G. H. Coles, \& G. D. Logan (Eds.), Converging operations in the study of visual selective attention (pp. 45-76). Washington, DC: American Psychological Association.

YANTIS, S., \& EGETH, H. E. (1994). Visual salience and stimulus-driven attentional capture [Abstract]. Investigative Ophthalmology \& Visual Science, 35, 1619.

YANTIS, S., \& JONIDES, J. (1984). Abrupt visual onsets and selective attention: Evidence from visual search. Journal of Experimental Psychology: Human Perception \& Performance, 10, 601-621.

\section{NOTE}

1. Although in the discussion of the previous experiment we expanded the conceptual definition of singleton to include both rows and columns, for purposes of exposition we will still distinguish singleton targets and distractors - those composed of a single element-from row and column targets and distractors.

(Manuscript received February 28, 1997; revision accepted for publication July $5,1997$. ) 